\title{
Design of Planar Wide Bandpass Filters from Wideband Planar Antennas
}

\author{
A. Abbosh $^{1}, \mathrm{M}$. Bialkowski ${ }^{1}$, and D. Thiel ${ }^{2}$ \\ ${ }^{1}$ School of ITEE, The University of Queensland, Q1d4072, Australia \\ \{abbosh,meb\}@itee.uq.edu.au \\ ${ }^{2}$ Griffith School of Engineering, Griffith University, Qld4111, Australia \\ d.thiel@griffith.edu.au
}

\begin{abstract}
Introduction
Wideband bandpass filters (BPF) are the key components of modern wideband communication systems. Due to the requirements of low cost and easy integration with the other circuit elements, planar filters have attracted most of the attention.

There are varieties of planar bandpass filters with wideband performance which have recently been proposed and investigated for use in wideband communication systems. For example, planar BPFs using end-coupled coplanar waveguides (CPW) were introduced in [1]. BPFs based on the combination of CPW lowpass and highpass periodic structures were described in [2]. In order to obtain tight coupling in a wideband BPF, the use of a parallel-coupled microstrip line with a slotted ground plane was postulated and investigated in [3]. An alternative approach to overcoming the manufacturing problems present in edge-coupled planar structures was demonstrated in [4], where broadsidecoupled structure was used to design ultra wideband BPFs.
\end{abstract}

Another type of wideband filters constructed by mounting a microstrip line in a lossy composite substrate so as to attenuate the signals at high frequencies was described in [5]. A wideband BPF employing two stopbands of a filter block with two tuning stubs on a ring was presented in [6]. A compact UWB bandpass filter using a single multimode resonator on microstrip line was reported in [7], whereas the design of a broadband BPF with a short-circuited CPW multimode resonator was given in [8].

In some BPF designs a particular attention is paid to improving the out-of-band rejection. Examples include a modified class of multimode resonator-based wideband filters with improved out of band rejection skirt [9] and harmonic passband suppression by employing electromagnetic bandgap periodic structures [10].

In this paper, we explore the idea of designing wideband BPFs by utilizing two identical wideband antennas. The tapered slot antenna is used for this purpose. In the proposed filter, two compact tapered slot antennas with CPW feedlines are connected in the faceto-face configuration to construct a BPF. The success of the proposed concept is demonstrated via full-wave electromagnetic simulations.

\section{Design}

The utilized antenna for the design of a wideband filter is shown in Fig. 1a. It belongs to a class of tapered slot antennas (TSA), which have attracted a significant attention due to their wideband performance, high directivity, low cost and easy integration with the radio frequency circuitry [11]. An inherent feature of TSA is that it has a natural low frequency cut-off. This cut-off frequency is determined by size of the radiating aperture which is approximately half-wavelength at that frequency. Otherwise, TSA has high-pass properties over ultra wide frequency band. Therefore to obtain a sharp high frequency cut-off, suitable means have to be devised. 
The TSA chosen in the present investigations has a linear slot profile. It is fed from a coplanar waveguide having broadband characteristics. The antenna's length $(l)$ and width $(w)$ are chosen following the guidelines presented in [11] and then optimised using the full-wave electromagnetic simulator CST Microwave Studio. To maintain a wide impedance bandwidth, the inner conductor of the feeder is tapered linearly in the manner shown in Fig. 1a. Assuming the use of Rogers RT6010 $\left(\varepsilon_{\mathrm{r}}=10.2\right.$, thickness $\left.=0.64 \mathrm{~mm}\right)$ as a substrate, dimensions of the antenna are: $L=15 \mathrm{~mm}$, and $W=30 \mathrm{~mm}$. For the $50 \Omega \mathrm{CPW}$ feeder, the dimensions are $W_{f}=2 \mathrm{~mm}$, and $S=0.5 \mathrm{~mm}$.

To form the proposed BPF, two identical TSA antennas are connected in the face-to-face orientation as depicted in Fig. 1b. The overall dimension of the filter is $3 \mathrm{~cm} \times 3 \mathrm{~cm}$. The input and output ports of the filter are coplanar waveguides.

In order to minimize the insertion loss of the designed filter at the passband, the structure shown in Fig. $1 b$ is enclosed in a metallic box. This configuration is used to prevent any possible radiation from the two antennas constituting the filter. Dimensions of the box are: width=length $=3 \mathrm{~cm}$ and height $=2 \mathrm{~cm}$. The filter's board is located at centre of the box.

\section{Results}

Performance of the proposed filter is verified using the commercial software package CST Microwave Studio.

Fig. 2 shows the simulated results for the return and insertion losses of the BPF. The results shown in Fig. 2 indicate that the device has a wide passband, which extends between $5.1 \mathrm{GHz}$ and $8.9 \mathrm{GHz}$ assuming the $3 \mathrm{~dB}$ insertion loss as a reference. It is worthwhile to mention here that the parametric analysis that we carried out indicates that the passband location and value can be controlled by the antenna's dimensions ( $L$ and $W$ ).

The insertion loss of the designed filters at the centre of the passband is less than $0.2 \mathrm{~dB}$, whereas the return loss is higher than $20 \mathrm{~dB}$. The low cut-off band (below $5 \mathrm{GHz}$ ) is sharp, providing $12 \mathrm{~dB} / \mathrm{GHz}$ roll-off. However, the high frequency band (especially above $11 \mathrm{GHz}$ ) has a slow cut-off rate. This is due to the high-pass behavior of the tapered slot antennas. To improve performance of the filter, the internal boundary of the structure is corrugated in the manner shown in Fig. 1b. Lengths of the used corrugations are chosen to be around half of the effective wavelengths at the high cut-off band (11 $\mathrm{GHz}$ ) to enable them to behave as a high band rejection filter. Dimensions of the corrugations were then optimized to achieve the best possible cut-off for the high band without jeopardizing the performance at the passband. The final values for the dimensions are: Length of the corrugations $=1 \mathrm{~mm}$ to $3 \mathrm{~mm}$, the width $=0.5 \mathrm{~mm}$ and the separation between them $=0.5 \mathrm{~mm}$. Performance of the modified device is shown in Fig. 2. It is apparent that the modified structure has a better high cut-off band with $20 \mathrm{~dB} /$ $\mathrm{GHz}$ roll-off, while the good performance at the passband and the low cut-off band is maintained. The passband for the corrugated structure extends between $4.8 \mathrm{GHz}$ and 8.5 GHz.

The simulated group delay for the designed filter (with corrugations) is depicted in Fig. 3. It is clear from this result that the device has a group delay which has a very low peak-topeak variation of less than $0.05 \mathrm{~ns}$ across the passband of the filter. Therefore, the designed filter shows attractive characteristics for wide BPF applications. 


\section{References}

[1] D. Williams, and S. Schwarz, "Design and performance of coplanar waveguide bandpass filters," IEEE Trans. Microwave Theory Tech., vol.31, pp.558-566, 1983.

[2] C. Hsu, F. Hsu, and J. Kuo, "Microstrip bandpass filters for ultra-wideband (UWB) wireless communications," IEEE MTT-S Int. Dig., pp. 679-682, 2005.

[3] M. Mandal ,and S. Sanyal, "Compact wideband bandpass filter," IEEE Microw. Wireless Compon. Lett., vol. 16, no. 1,pp.46-48, 2006.

[4] A. Abbosh, "Planar bandpass filters for ultra wideband applications," IEEE Trans. Microwave Theory Tech., vol. 55, no. 10, pp. 2262-2269, 2007.

[5] A. Saito, H. Harada, and A. Nishikata, "Development of band pass filter for ultra wideband (UWB) communication," Proc. IEEE Conf. Ultra Wideband Systems Technology, pp. 76-80, 2003.

[6] L. Hsieh, and K. Chang, "Compact, low insertion-loss, sharp-rejection, and wide-band microstrip bandpass filters," IEEE Trans. Microwave Theory Tech., vol. 51, no. 4, pp. 1241-1246, 2003.

[7] L. Zhu, S. Sun, and W. Menzel, "Ultra-wideband (UWB) bandpass filters using multiple-mode resonator," IEEE Microw. Wireless Compon. Lett., vol. 15, no. 11, pp. 796-798, 2005.

[8] J. Gao, L. Zhu, W. Menzel, and F. Bögelsack, "Short-circuited CPW multiplemode resonator for ultra-wideband (UWB) bandpass filter," IEEE Microwave Wireless Compon. Lett., vol.16, no.3, 104-106, 2006.

[9] N. Thomson, and J. Hong, "Compact ultra-wideband microstrip/coplanar waveguide bandpass filter," IEEE Microw. Wireless Compon. Lett., vol. 17, no.3, pp.14-186, 2007.

[10] J. García-García, J. Bonache, and F. Martín, "Application of electromagnetic bandgaps to the design of ultra-wide bandpass flters with good out-of-band performance," IEEE Trans. Microwave Theory Tech., vol. 54, no. 12, pp. 4136-4140, 2006.

[11] A. Abbosh, M. Bialkowski and H. Kan, Planar tapered slot antennas, Chapter six in Printed Antennas for Wireless Communications Handbook, edited by R. Waterhouse, Wiley Int., USA, 2007.

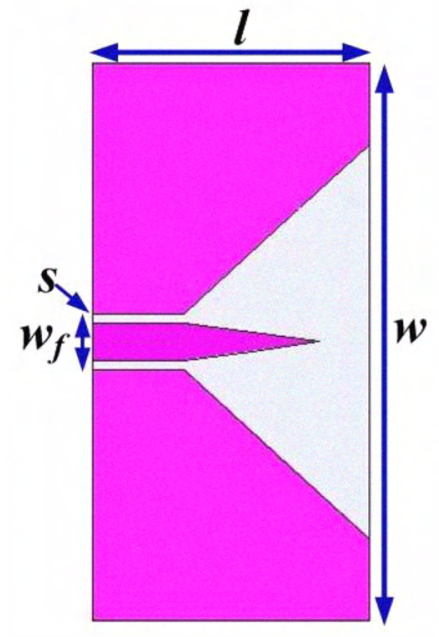

(a)

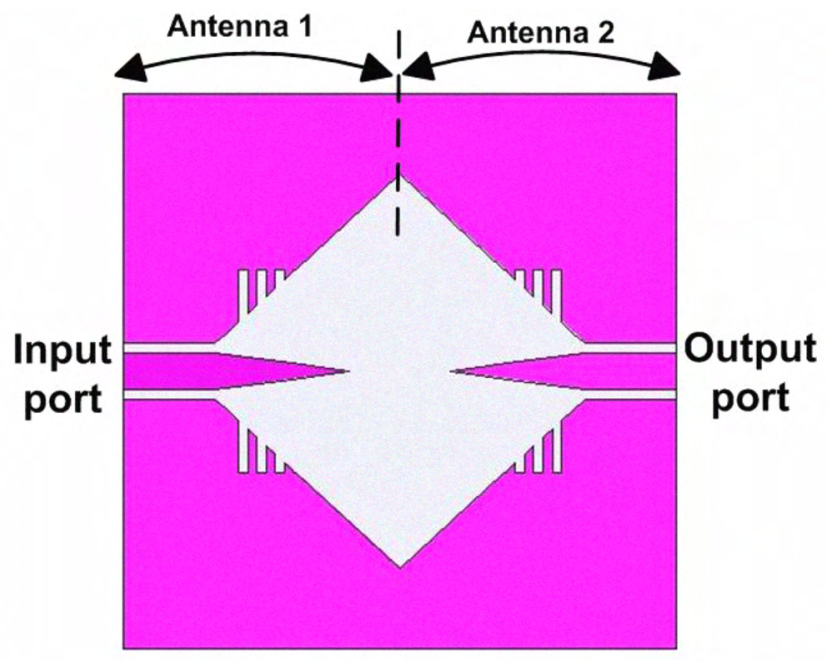

(b)

Fig.1 The tapered slot antenna (a), and the filter composed of two corrugated antennas (b). 


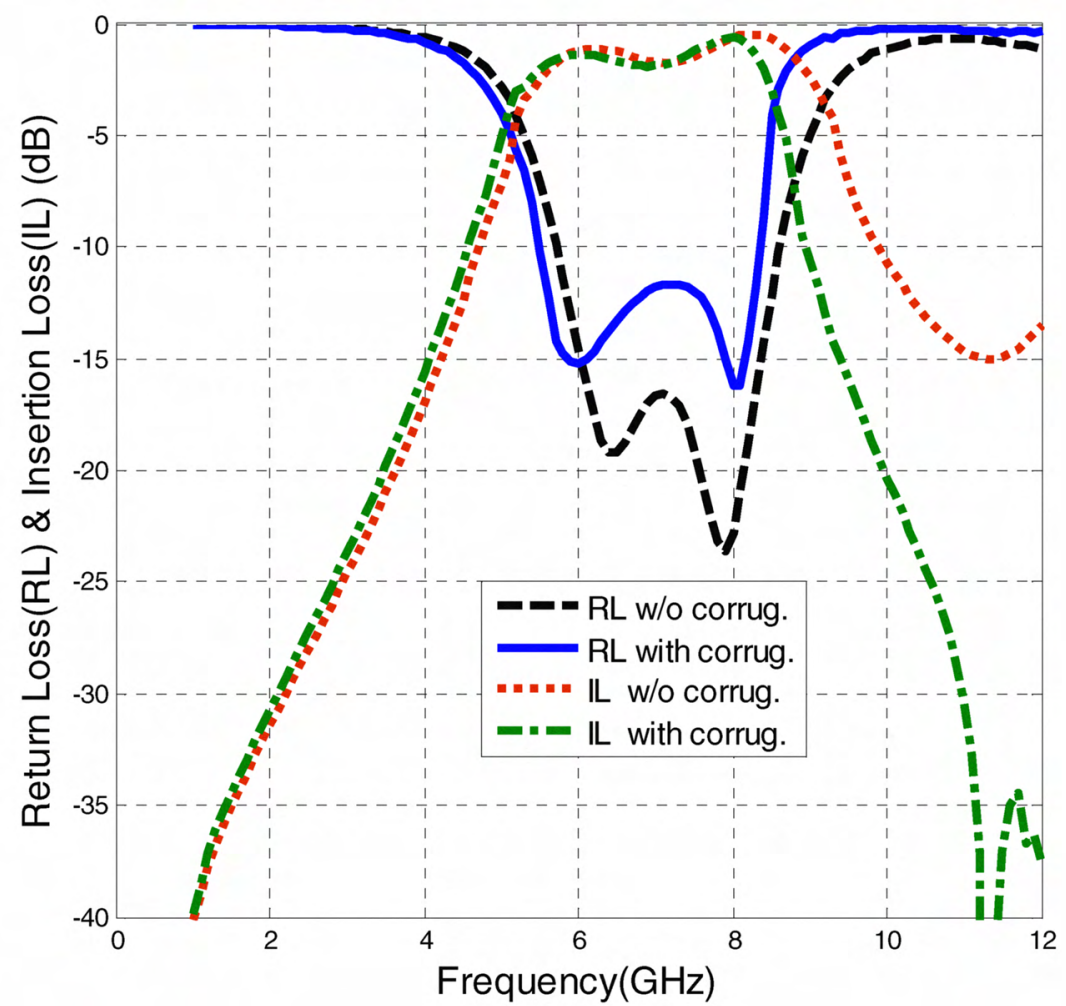

Fig. 2 Variation of the return loss (RL) and insertion loss (IL) with frequency for the structure with and without corrugations.

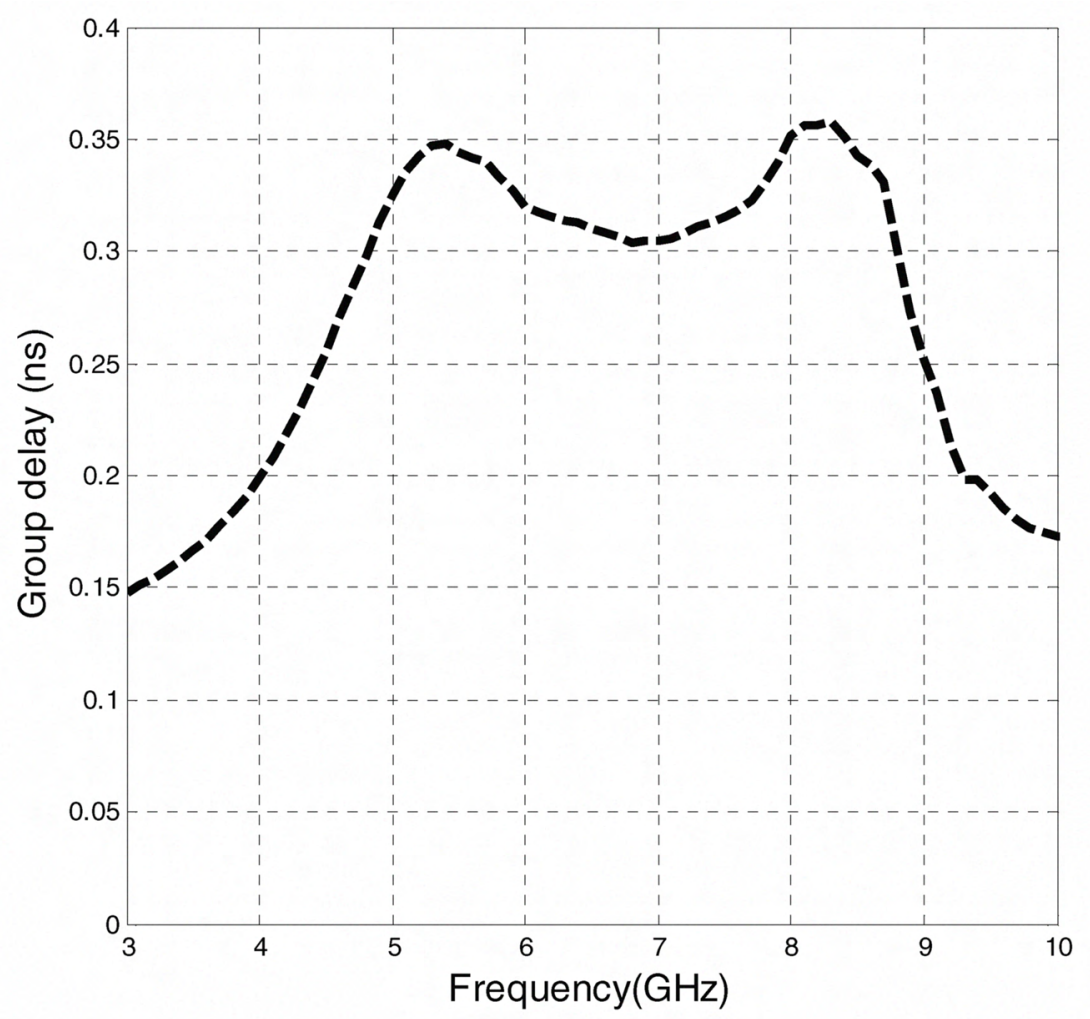

Fig. 3 Variation of the group delay with frequency for the structure shown in Fig. 1b. 